\title{
Molecular evolution of antibody cross-reactivity for two subtypes of type A botulinum neurotoxin
}

\author{
Consuelo Garcia-Rodriguez ${ }^{1,3}$, Raphael Levy ${ }^{1,3}$, Joseph W Arndt ${ }^{2}$, Charles M Forsyth ${ }^{1}$, Ali Razai ${ }^{1}$, \\ Jianlong Lou ${ }^{1}$, Isin Geren ${ }^{1}$, Raymond C Stevens ${ }^{2}$ \& James D Marks ${ }^{1}$
}

Broadening antibody specificity without compromising affinity should facilitate detection and neutralization of toxin and viral subtypes. We used yeast display and a co-selection strategy to increase cross-reactivity of a single chain (sc) Fv antibody to botulinum neurotoxin type $A$ (BoNT/A). Starting with a ScFv that binds the BoNT/A1 subtype with high affinity (136 pM) and the BoNT/A2 subtype with low affinity (109 nM), we increased its affinity for BoNT/A2 1,250-fold, to $87 \mathrm{pM}$, while maintaining high-affinity binding to BoNT/A1 (115 pM). To find the molecular basis for improved cross-reactivity, we determined the X-ray co-crystal structures of wild-type and cross-reactive antibodies complexed to BoNT/A1 at resolutions up to $2.6 \AA$, and measured the thermodynamic contribution of BoNT/A1 and A2 amino acids to wild-type and cross-reactive antibody binding. The results show how an antibody can be engineered to bind two different antigens despite structural differences in the antigen-antibody interface and may provide a general strategy for tuning antibody specificity and cross-reactivity.

Botulism is caused by botulinum neurotoxin (BoNT) and is characterized by flaccid paralysis, which if not immediately fatal requires prolonged hospitalization in an intensive care unit. Naturally occurring botulism results from ingestion of contaminated food, anaerobic wound infections or gastrointestinal tract colonization by clostridial bacteria ${ }^{1}$. BoNTs are also classified by the Centers for Disease Control as one of the six highest-risk threat agents for bioterrorism (the 'Category A agents'), owing to their extreme potency and lethality ${ }^{2-4}$.

Treatment of botulism in adults requires use of antitoxin ${ }^{5}$ generated from immunized horses, which is associated with a high incidence of side effects, including serum sickness and anaphylactic shock ${ }^{6}$. As an alternative, monoclonal antibodies (mAbs) are under development ${ }^{7,8}$. Development of a mAb-based antitoxin for botulism is complicated by two factors. First, single mAbs, regardless of affinity, have not neutralized toxin with a potency that would allow practical development; however combinations of two to three mAbs neutralize BoNT with high potency ${ }^{8}$. Second, there exist multiple subtypes for each of the seven toxin serotypes, which differ from each other by up to $35 \%$ at the amino acid level. These subtype sequence differences affect mAb binding and neutralization ${ }^{9}$. For example, the amino acid sequences of BoNT subtype A2 (BoNT/A2 (ref. 10)) and BoNT subtype A1 (BoNT/ A1 (ref. 11)) differ by $10 \%$ (ref. 9). As a result, only three of six mAbs bind both toxin subtypes with high affinity ${ }^{9}$. The humanized affinitymatured $\mathrm{mAb}$ AR2 binds BoNT/A1 with very high affinity $\left(K_{\mathrm{D}}=6.8\right.$ $\left.\times 10^{-12} \mathrm{M}\right)$ and exhibits the highest level of in vivo neutralization we have observed for a single $m \mathrm{Ab}^{12}$. Unfortunately, AR2 IgG binds BoNT/A2 with more than a 29,000-fold lower affinity than BoNT/A1, and as a result has minimal neutralizing activity for BoNT/A2 (ref. 9).
To overcome these challenges, we used yeast display, antibody gene diversity libraries, and a dual-selection strategy to increase antibody affinity for BoNT/A2 more than 1,000-fold while maintaining high affinity binding to BoNT/A1. Measurement of the thermodynamics of antibody binding to BoNT/A1 and BoNT/A2 and solution of the $\mathrm{X}$-ray co-crystal structure of antibodies complexed to toxin reveal the basis for increased cross-reactivity and suggest a general route to broadening antibody specificity.

\section{RESULTS}

Improving the cross-reactivity of AR2 for BoNT/A1 and BoNT/A2 A gene diversity library was used to increase the affinity of the previously created AR2 $\mathrm{scFv}^{12}$ for BoNT/A2 while maintaining highaffinity binding to BoNT/A1. An AR2 scFv-based yeast display library was generated by using a spiked oligonucleotide to introduce mutations into the heavy-chain variable region $\left(\mathrm{V}_{\mathrm{H}}\right)$ antigen-binding loop H1 (Table 1) ${ }^{13,14}$. Loop amino acids were selected for mutagenesis based on solvent accessibility as determined by modeling. In $\mathrm{H} 1$, the loop residues begin in the framework region (as classified by Kabat ${ }^{13}$ ) and extend into complementarity determining region 1 (CDR1).

For selection of a cross-reactive scFv, the induced library was initially flow sorted after staining with BoNT/A1. The next four rounds of sorting were done with decreasing concentrations of BoNT/A2. After five rounds of sorting, the gene encoding the $\mathrm{ScFv}$ was amplified from individual colonies, revealing the presence of one unique sequence, CR1 (Table 1). Compared to AR2, CR1 had three amino acid substitutions in $\mathrm{H} 1$ and a single amino acid substitution in $\mathrm{H} 2$ (P61S) (Table 1). The affinity of yeast-displayed CR1 scFv for

\footnotetext{
${ }^{1}$ Department of Anesthesia and Pharmaceutical Chemistry, University of California, San Francisco Rm. 3C-38, San Francisco General Hospital, 1001 Potrero Ave., San Francisco, California 94110, USA. 2Department of Molecular Biology, The Scripps Research Institute, 10550 N. Torrey Pines Road, La Jolla, California 92037, USA.

${ }^{3}$ These authors contributed equally to this work. Correspondence should be addressed to J.D.M. (marksj@anesthesia.ucsf.edu).
} 
Table 1 Sequences, affinity and binding kinetics of wild-type and cross-reactive antibodies to BoNT/A1 and BoNT/A2

\begin{tabular}{|c|c|c|c|c|c|c|c|}
\hline \multicolumn{8}{|c|}{ Sequences and affinities of yeast-displayed scFv } \\
\hline Clone & $\mathrm{H} 1$ & $\mathrm{H} 2$ & H3 & L1 & $K_{\mathrm{D}} \mathrm{A} 1(\mathrm{nM})$ & $K_{\mathrm{D}} \mathrm{A} 2(\mathrm{nM})$ & $K_{D} A 2 / K_{D} A 1$ \\
\hline AR2 & ${ }^{28} \mathrm{TFSDH}^{32}$ & ${ }^{53}$ DGGSY57 & ${ }^{99}$ YRYDD $^{103}$ & ${ }^{28}$ SVDSY32 & 0.136 & 109 & 801 \\
\hline CR1 & $--K Y D$ & ----- & ----- & ----- & 0.125 & 0.4 & 3.2 \\
\hline CR $1.1^{\mathrm{a}}$ & $--K Y D$ & ----- & ----- & ----- & 0.087 & 0.106 & 1.2 \\
\hline $\mathrm{CR} 1.2^{\mathrm{b}}$ & $--K Y D$ & ----- & ----- & ----- & 0.082 & 0.182 & 2.2 \\
\hline $\mathrm{CR} 2^{\mathrm{C}}$ & $--K Y D$ & ----- & ----- & ----- & 0.115 & 0.087 & 0.76 \\
\hline
\end{tabular}

Affinities and binding kinetics of purified IgG

\begin{tabular}{|c|c|c|c|c|c|c|}
\hline \multirow[b]{2}{*}{ Antibody } & \multicolumn{3}{|c|}{ BoNT/A1 } & \multicolumn{3}{|c|}{ BoNT/A2 } \\
\hline & $K_{\mathrm{D}}\left(\mathrm{M}^{-1}\right)$ & $k_{\mathrm{on}}\left(\mathrm{M}^{-1} \mathrm{~s}^{-1}\right)$ & $k_{\mathrm{off}}\left(\mathrm{s}^{-1}\right)$ & $K_{\mathrm{D}}\left(\mathrm{M}^{-1}\right)$ & $k_{\mathrm{on}}\left(\mathrm{M}^{-1} \mathrm{~s}^{-1}\right)$ & $k_{\mathrm{off}}\left(\mathrm{s}^{-1}\right)$ \\
\hline AR2 IgG & $6.8 \times 10^{-12}$ & $3.70 \times 10^{6}$ & $2.51 \times 10^{-5}$ & $2.01 \times 10^{-7}$ & $8.31 \times 10^{5}$ & $1.67 \times 10^{-1}$ \\
\hline CR1 IgG & $2.48 \times 10^{-12}$ & $3.61 \times 10^{6}$ & $8.96 \times 10^{-6}$ & $1.73 \times 10^{-9}$ & $1.62 \times 10^{7}$ & $2.81 \times 10^{-2}$ \\
\hline CR2 IgG & $1.01 \times 10^{-11}$ & $3.56 \times 10^{6}$ & $3.56 \times 10^{-5}$ & $2.9 \times 10^{-10}$ & $1.58 \times 10^{7}$ & $4.57 \times 10^{-3}$ \\
\hline
\end{tabular}

BoNT/A1 was essentially unchanged compared to AR2 $\left(1.36 \times 10^{-10}\right.$ $\mathrm{M}$ versus $1.25 \times 10^{-10} \mathrm{M}$, respectively), whereas its affinity for BoNT/ A2 increased 272-fold, from $1.09 \times 10^{-7} \mathrm{M}$ to $4.0 \times 10^{-10} \mathrm{M}$ (Table 1). Mutation of the P61S mutation in the H2 of CR1 back to P61 resulted in no change in affinity for BoNT/A1 and a less than twofold reduction in affinity for BoNT/A2, indicating that the increase in cross-reactivity was due almost exclusively to the $\mathrm{H} 1$ mutations.

Evolution of CR1 to increase cross-reactivity for BoNT/A1 and A2 To further improve the affinity of the CR1 scFv for BoNT/A2 while maintaining high-affinity binding to BoNT/A1, we generated three additional yeast display libraries based on the sequence of CR1. These libraries were generated by using spiked oligonucleotides to introduce mutations into the $\mathrm{H} 2, \mathrm{H} 3$ or L1 antigen-binding loops (Table 1). For selection of cross-reactive scFv, the induced libraries were stained with BoNT/A1 for the first and fourth rounds of sorting and stained with BoNT/A2 for the second and third rounds of sorting. For subsequent sorting rounds, yeast were simultaneously incubated with BoNT/A2 holotoxin and the receptor binding domain $\left(\mathrm{H}_{\mathrm{C}}\right)$ of BoNT/A1 using a labeling strategy that provided independent sortable signals for BoNT/

Figure 1 Use of flow cytometry to select for yeast-displayed scFv binding both BoNT/A1 and BoNT/A2. Representative dot plots showing the method for selection of cross-reactive yeast-displayed scFv. $(\mathbf{a}, \mathbf{b})$ For the first through fourth rounds of sorting (second and third rounds shown here), yeast were stained with decreasing concentrations of either BoNT/A1 or BoNT/A2 (labeled as described in the text to generate fluorescence in the APC channel) and SV5-488 (FITC channel) to quantify the scFv display level. Yeast with the highest mean fluorescence intensity for BoNT/A binding were gated and sorted (gate P2). APC, allophycocyanin; FITC, fluorescein isothiocyanate. (c) For the fifth and later rounds of sorting, yeast were labeled with BoNT/A1 and BoNT/A2 toxins in a manner permitting independent quantification of the extent of BoNT/A1 binding (PE channel) and BoNT/A2 binding (APC channel). Yeast with the highest mean fluorescence intensity for BoNT/A1 binding (gate P3) and BoNT/A2 binding (gate P2) were gated, the gates intersected and only yeast in both gates sorted. PE, phycoerythrin.
A1 and BoNT/A2 binding (Fig. 1). To select yeast displaying scFv with the highest-affinity binding to both BoNT/A subtypes, yeast with the highest mean fluorescence intensity for binding to BoNT/A1 and BoNT/A2 were separately gated and only yeast present in both gates sorted by intersecting the two gates (Fig. 1c).

After six rounds of sorting, the gene encoding the scFv was amplified from individual colonies for each of the three libraries. Overall, it proved difficult to further increase the affinity of CR1 for BoNT/A2 while maintaining high-affinity binding to BoNT/A1. The only scFv identified with higher affinity for BoNT/A2 and no reduction in affinity for BoNT/A1 had wild-type CDRs and mutations in the framework regions, owing to PCR errors. CR1.1 had a single mutation located outside of H2, V37I, and an $\sim 50 \%$ improvement in affinity for BoNT/A1 and a fourfold increase in affinity for BoNT/A2 (Table 1). CR1.2 had a single
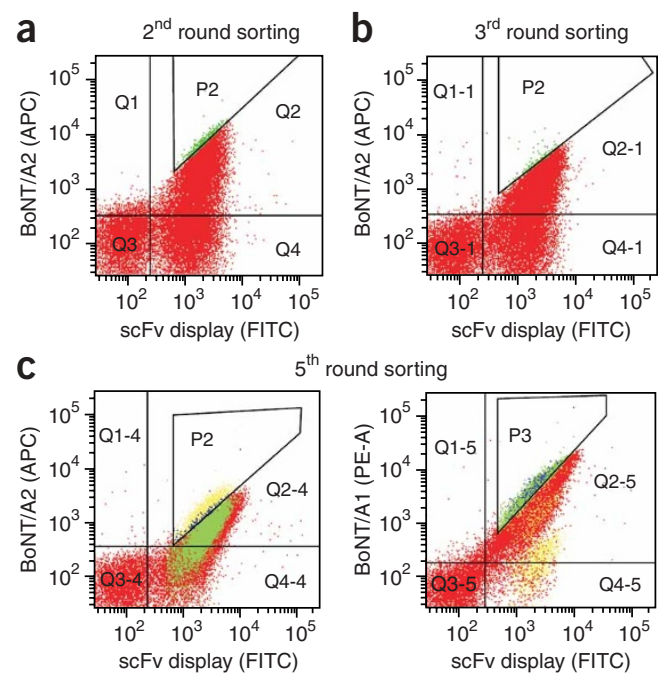

$5^{\text {th }}$ round sorting

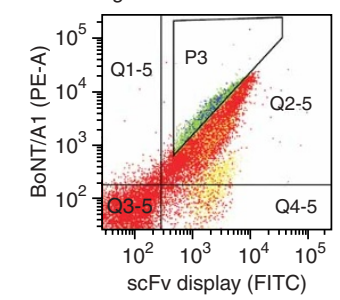


a

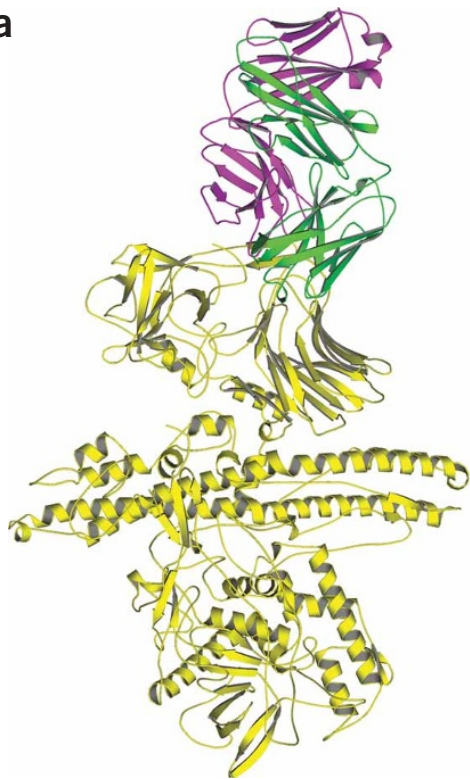

b
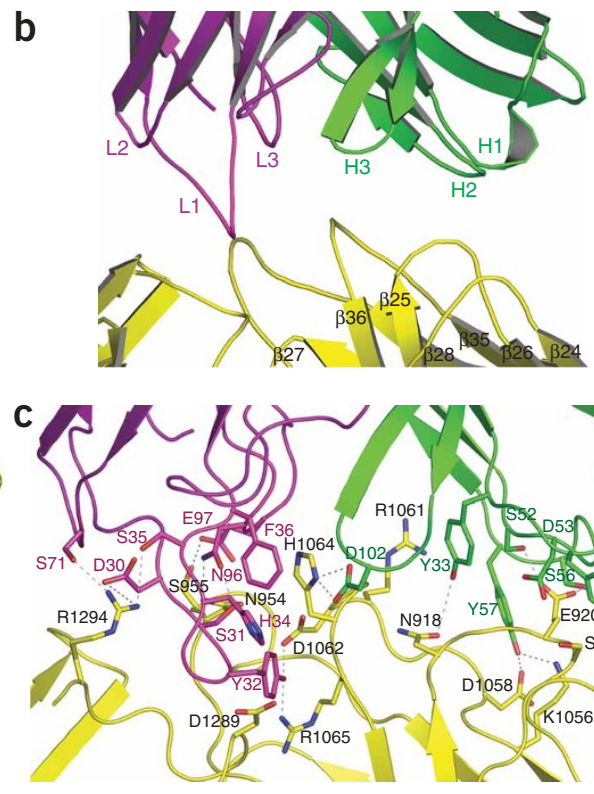

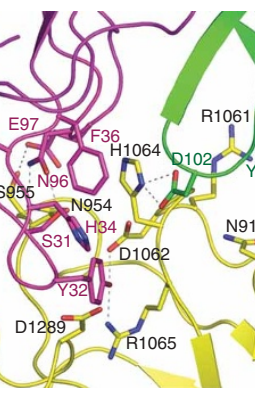

Figure 2 Overview and specific interactions of the CR1-BoNT/A1 co-crystal. (a) Overall view of BoNT/A1 (yellow) in complex with the CR1 Fab with its light and heavy chains in magenta and green, respectively. (b) Overview of the CR1-BoNT/A1 interface, with the antigen contacting loops ( $\mathrm{H} 1, \mathrm{H} 2$, H3, L1, L2 and L3) and toxin $\beta$-strands indicated. (c) Detailed view of contacts between CR1 Fab and BoNT/A1. A cartoon representation of BoNT/A1 is shown with carbons (yellow), nitrogens (blue) and oxygens (red). Amino acid contacts are indicated by magenta $\left(V_{L}\right)$, green $\left(V_{H}\right)$ and black (BoNT/A) o) numbering. $V_{L}$, variable light chain; $V_{H}$, variable heavy chain. respectively, for the $\mathrm{scFv}$ ), while maintaining high-affinity binding to BoNT/A1 (Table 1 and Supplementary Fig. 1 online). As the $K_{\mathrm{D}}$ s of both AR2 and CR2 IgG for BoNT/A1 were 10- to 20 -fold higher than the $\mathrm{scFv} K_{\mathrm{D}} \mathrm{S}$, but the IgG and $s c F v K_{\mathrm{D}}$ f for BoNT/A2 were comparable, the relative $\operatorname{IgG}$ affinity for BoNT/A1 versus BoNT/A2 decreased from 29,000-fold to 29-fold (a 1,000-fold narrowing of relative affinities) compared to 801fold to 0.76-fold for the scFv (a 1,054-fold narrowing of relative affinities). Thus, although the absolute differences in relative $K_{\mathrm{D}}$ were different between the IgG and scFv format, the relative increase in affinity for BoNT/A2 versus BoNT/A1 was translatable from the $s c F v$ format to the IgG. The differences in absolute $K_{\mathrm{D}}$ between $\mathrm{scFv}$ and IgG may reflect differences in scFv stability compared to IgG (usually higher for IgG), the fact that scFvs were displayed on yeast and the IgG were in solution, or differences in techniques used to measure the $K_{\mathrm{D}}$. The difference does not appear to be due to valency, as the $K_{\mathrm{D}} \mathrm{S}$ of CR1 Fab and IgG for BoNT/A1 and BoNT/A2 are comparable when measured by the same technique $\left(\mathrm{CR} 1 \mathrm{Fab} K_{\mathrm{D}}\right.$ $=5.0 \times 10^{-12} \mathrm{M}$ and $2.0 \times 10^{-9} \mathrm{M}$ for BoNT/A1 and BoNT/A2, respectively). substitution $\mathrm{V}_{\mathrm{H}}$ E6Q that resulted in an $\sim 50 \%$ improvement in affinity for BoNT/A1 and a twofold increase in affinity for BoNT/A2. The $V_{H}$ V37I mutation of clone CR1.1 was combined with the $V_{H}$ E6Q mutation of clone CR1.2 to yield the double mutant CR2, which had a 4.5 -fold increase in affinity for BoNT/A2 and no change in affinity for BoNT/A1 (Table 1).

Using the co-crystal structure of CR1-BoNT/A1 (see below), nine mutants of CR1 were also generated to either increase hydrogen bonding or van der Waals interaction at the periphery of the interface, increase surface complementarity at the center of the interface or increase hydrophobic interactions. None of these mutations increased cross-reactivity, and most decreased affinity for BoNT/A2 (Supplementary Table 1 online). These results illustrate the challenge in increasing antibody cross-reactivity, even with a co-crystal structure, and highlight the combined power of the diversity library and selection approaches used here.

Overall, the evolution of AR2 to CR2 resulted in a scFv that had the same affinity for BoNT/A1 but whose affinity for BoNT/A2 increased 1,250 -fold, from $1.09 \times 10^{-7}$ to $8.7 \times 10^{-11} \mathrm{M}$. This narrowed the relative affinities for BoNT/A2:BoNT/A1 from 800-fold for AR2 to no difference for CR2. The six mutations in AR2 accounting for this increase in cross-reactivity are all located in the $\mathrm{V}_{\mathrm{H}}$ domain, with three mutations in solvent-accessible residues of the $\mathrm{V}_{\mathrm{H}} \mathrm{H} 1$.

\section{Affinities of IgG constructed from ScFv}

To determine whether changes in affinity observed for the scFvs were recapitulated in the IgGs, we converted CR1 and CR2 scFv to IgG. As with the scFv, CR1 and CR2 IgG showed substantially increased affinity for BoNT/A2 compared to the parental AR2 (116-fold and 693-fold, respectively, for IgG compared to 272-fold and 1,252-fold,

\section{Structural and functional epitope of AR2 and CR1}

To understand the molecular basis of engineered cross-reactivity, we crystallized BoNT/A1 holotoxin in complex with the CR1 and AR2 Fabs. Both Fabs bind to identical regions on the BoNT/A1 binding domain, at the interface between the N-terminal lectin subdomain $\left(\mathrm{H}_{\mathrm{CN}}\right)$ and the C-terminal trefoil subdomain $\left(\mathrm{H}_{\mathrm{CC}}\right)$ (Fig. 2). The BoNT/A1-CR1 structure has a discontinuous epitope that is predominantly composed of the $\beta 23-\beta 24, \beta 25-\beta 26, \beta 27-\beta 28$ and $\beta 35-\beta 36$ loops of the lectin subdomain, but also includes the $C$ terminus of the trefoil (Fig. 2a,b and Table 2). The buried interface surface area of CR1 is $1,126 \AA^{2}$, with nearly an equal contribution being made by the CR1 heavy $\left(588 \AA^{2}\right)$ and light $\left(538 \AA^{2}\right)$ chains. Compared with the structure of the uncomplexed BoNT/A1, the trefoil subdomain is displaced 7.5 $\AA$ after antibody binding, and several BoNT loops are repositioned to enable optimal binding to $\mathrm{CR} 1$ residues.

Interactions of the CR1 light chain with BoNT/A1 are found in all three of the antigen-binding loops (L1-L3), but are primarily restricted to L1 and L3. L1 binds the $\beta 35-\beta 36$ loop of BoNT/A1; key interactions include strong hydrogen bonding between Y32 of CR1 and the side chains of D1062 and R1065 of BoNT/A1 and pistacking interactions of F36 of CR1 with H1064. L3 binds via G95 and N96 also forms a hydrogen bond with H1064 of BoNT/A1, whereas E97 interacts with S955 in the $\beta 27-\beta 28$ loop of BoNT/A1. Only a single contact is found in $\mathrm{L} 2$ with the $\mathrm{C}$ terminus of the trefoil domain via S71 of CR1 with R1294 of BoNT/A1 (Fig. 2b,c and Table 2).

Interactions of the CR1 heavy chain with BoNT/A1 are also found in all three loops $(\mathrm{H} 1-\mathrm{H} 3)$. The H1 of CR1 has a variety of side-chain and main-chain interactions, with Y31 and Y33 forming hydrogen bonds to S902 and N918 of BoNT/A1 (Fig. 2b,c and Table 2). The dominant contribution of the CR1 heavy chain in BoNT/A1 binding is from CDR $\mathrm{H} 2$ of CR1. In H2, the D53 side-chain hydrogen bonds 
Table 2 Direct contacts between CR1 Fab and BoNT/A1 and the energetics of AR2 and CR1 binding to BoNT/A1 and BoNT/A2

\begin{tabular}{|c|c|c|c|c|c|}
\hline \multicolumn{2}{|c|}{ Van der Waals contacts, hydrogen bonds and salt bridges } & \multicolumn{4}{|c|}{ Energetics of toxin side chain binding to Fab $\Delta \Delta G$ upon side chain mutation to alanine } \\
\hline BoNT/A1 residue/atom & CR1 residue/atom distance $(\AA)$ & AR2 Fab BoNT/A1 & CR1 Fab BoNT/A1 & AR2 Fab BoNT/A2 & CR1 Fab BoNT/A2 \\
\hline Ser $902 /$ Asp OG & Y31H OH/3.68 ^ & -0.12 & -0.23 & -0.49 & -0.18 \\
\hline Lys 903/Arg & No contact & 0.54 & 0.29 & -0.02 & 1.01 \\
\hline Gln 915/Lys & No contact & 0.10 & 0.52 & -0.31 & -0.32 \\
\hline Phe $917 / l l e$ & $\mathrm{Y} 101 \mathrm{H}$ & -0.05 & 0.42 & 0.95 & -0.49 \\
\hline Asn 9180 & Y33H OH/3.25 & 2.16 & 0.89 & 0.30 & -1.4 \\
\hline Leu 919 & Y33H, Y101H & 2.28 & 2.59 & 0.41 & 0.57 \\
\hline Glu 920 & $\mathrm{~S} 52 \mathrm{H}, \mathrm{G} 54 \mathrm{H}, \mathrm{S} 56 \mathrm{H}, \mathrm{Y} 57 \mathrm{H}$ & 2.77 & 2.84 & 0.50 & 1.74 \\
\hline Glu 920 N & D53H 0D1/3.06 ^ & - & - & - & - \\
\hline & D53H 0D2/3.05 & - & - & - & - \\
\hline Glu 920 OE1 & $\mathrm{S} 52 \mathrm{H}$ OG/2.56 § & - & - & - & - \\
\hline & $\mathrm{S} 56 \mathrm{H} \mathrm{N} / 3.41 \AA$ & - & - & - & - \\
\hline & G54H N/3.03 & - & - & - & - \\
\hline Lys 923/Thr & No contact & -0.30 & -0.14 & 0.62 & -0.29 \\
\hline Phe 953 & Y32L & 3.34 & 4.06 & 1.53 & 2.64 \\
\hline Asn 954/Ser & E97L & -0.15 & 0.10 & -0.66 & 0.13 \\
\hline Asn 954 OD1 & N96L ND2/3.00 ̊ & - & - & - & - \\
\hline Ser 955 OG/Lys & E97L OE2/2.77 ̊ & -0.08 & 0 & -0.5 & -0.55 \\
\hline & E97L OE1/3.09 $\AA$ & - & - & - & - \\
\hline Ile 956 & E97L & 0.07 & -0.01 & ND & ND \\
\hline Ser 957/Asn & No contact & - & - & 0.008 & 0.2 \\
\hline Lys 1056 & $\mathrm{Y} 57 \mathrm{H}$ & -0.01 & -0.03 & ND & ND \\
\hline Lys 1056 NZ & Y57H OH/3.41 & - & - & - & - \\
\hline Asp 1058 OD1 & Y57H OH/2.57 ̊ & -0.03 & 0.01 & ND & ND \\
\hline Arg 1061 & $\mathrm{Y} 59 \mathrm{H}$ & 0.29 & 0.82 & 0.58 & 0.70 \\
\hline Arg $1061 \mathrm{NH} 1$ & $\mathrm{~T} 58 \mathrm{H} 0 / 3.20 \AA$ & - & - & - & - \\
\hline Arg $1061 \mathrm{NH} 2$ & T58H 0/2.87 ̊̊ & - & - & - & - \\
\hline Asp 1062 OD1 & Y32L OH/2.56 ̊ & 2.34 & 2.53 & -0.14 & -0.10 \\
\hline Thr 1063 /Pro & F100L, Y101H & 2.37 & 1.62 & 0.24 & 0.93 \\
\hline His 1064 /Arg & Y32L, G95L, F36L & 7.42 & 7.32 & 4.44 & 4.91 \\
\hline His 1064 ND1 & $\mathrm{D} 102 \mathrm{H} 0 \mathrm{D} 1 / 2.88 \AA$ & - & - & - & - \\
\hline & D102H OD2/2.73 & - & - & - & - \\
\hline His 1064 NE2 & N96L 0/3.42 ̊ & - & - & - & - \\
\hline & G95L 0/2.68 ^ & - & - & - & - \\
\hline Arg 1065 & Y32L & ND & ND & ND & ND \\
\hline Arg $1065 \mathrm{NH} 1$ & Y32L OH/2.77 & - & - & - & - \\
\hline Gly 1292 & G33L, S71L & ND & ND & ND & ND \\
\hline Arg $1294 /$ Ser NH2 & S35L OG/3.29 ̊ & 0.39 & 0.30 & ND & ND \\
\hline
\end{tabular}

BoNT residues in and near the structural epitope of the CR1-BoNT/A1 complex are listed, with the type of contact indicated. BoNT/A1 and CR1 atoms and distances are listed for hydrogen bonds and salt bridges. The seven BoNT/A1 amino acids that differ from BoNT/A2 and that have direct contacts with CR1 are bold. The contribution of BoNT/A1 and BoNT/A2 amino acid side chains to the energetics of binding to AR2 or CR1 Fab were determined by mutating each amino acid to alanine, measuring the $K_{\mathrm{D}}$ of wild-type and mutant toxin for the Fab, and calculating the $\Delta \Delta \mathrm{G}$ (see text for details). The $\Delta \Delta \mathrm{G}$ of the seven amino acids with substantial energetic contribution to binding of BoNT/A1 to AR2 $(\Delta \Delta \mathrm{G}>1.0)$ are bold. ND, not determined.

with the amide nitrogens of E920 and S921 of the $\beta 25-\beta 26$ loop of BoNT/A1, whereas the E920 side chain interacts with S52 and S56 of $\mathrm{CR} 1$. The $\mathrm{H} 2$ also interacts with the $\beta 27-\beta 28$ loop of BoNT/A1 with hydrogen bonds between T58 and Y57 of CR1 with K1056, D1058, G1059 and R1061 of BoNT/A1 (Fig. 2b,c and Table 2). In addition, the $\mathrm{H} 3$ of $\mathrm{CR} 1$ contributes to the binding with a hydrogen bond between D102 of CR1 with the H1064 of BoNT/A1 and van der Waals contacts between Y101 of CR1 with F917, L919 and T1063 of BoNT/A1.

To determine the functional epitopes of AR2 and CR1, BoNT/A1 $\mathrm{H}_{\mathrm{C}}$ was displayed on the surface of yeast. A 'BoNT/A2' $\mathrm{H}_{\mathrm{C}}$ was also displayed on yeast by mutating all seven BoNT/A1 amino acids in the structural epitope, as well as three nearby residues, K903, Q915 and K923, to the BoNT/A2 residue. The affinity of AR2 and CR1 Fab for yeast-displayed BoNT/A1 and BoNT/A2 $\mathrm{H}_{\mathrm{C}}$ was comparable to the solution $K_{\mathrm{D}}$ of their respective IgG for BoNT/A1 and BoNT/A2 (CR1 Fab $K_{\mathrm{D}}=43 \mathrm{pM}$ and $2.5 \mathrm{nM}$ for BoNT/A1 and BoNT/A2 yeast displayed; $2.5 \mathrm{pM}$ and $1.73 \mathrm{nM}$ for IgG in solution: AR2 Fab $K_{\mathrm{D}}=73$ $\mathrm{pM}$ and $100 \mathrm{nM}$ for BoNT/A1 and BoNT/A2 yeast displayed; $6.8 \mathrm{pM}$ and $201 \mathrm{nM}$ for IgG in solution). Each amino acid side chain in the structural epitope of BoNT/A1 or BoNT/A2 was truncated by mutation to alanine, the $K_{\mathrm{D}}$ of the yeast-displayed alanine mutant measured for $\mathrm{CR} 1$ and $\mathrm{AR} 2 \mathrm{Fab}$, and the values used to determine the $\Delta \Delta \mathrm{G}$.

The functional epitopes of AR2 and CR1 for BoNT/A1 were virtually identical (Table 2) and were smaller than the structural epitope, with only seven toxin side chains having energetically important interactions with the antibodies (N918, L919, E920, F953, D1062, T1063 and H1064). The most energetically important interaction is with H1064; truncation of the H1064 side chain to alanine 
a

a BoNT/A1
BoNT/A2

BoNT/A1

BoNT/A1
BoNT/A2

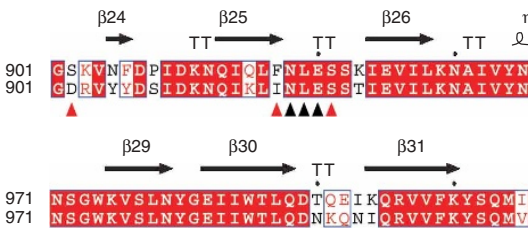

$\eta 14$

$\beta 27$

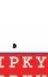

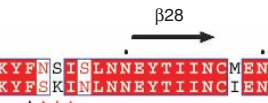
$\triangle \Delta \Delta$$$
\text { TT }
$$

$\begin{array}{ll}\text { BoNT/A1 } & 1041 \\ \text { BoNT/A2 } & 1041\end{array}$

TT

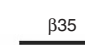

TT $\beta 36$

TT $\beta 32$

TT

$\stackrel{\beta 33}{\longrightarrow} \mathrm{TT} \stackrel{\beta 34}{\longrightarrow}$

ISDYINRWIFVTITNNRINNSRIYINGRLIDQRP
I SDYINRWIFVTITINARLTKSKIYINGRLIDQKP (1)

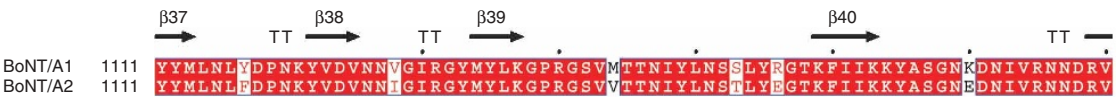
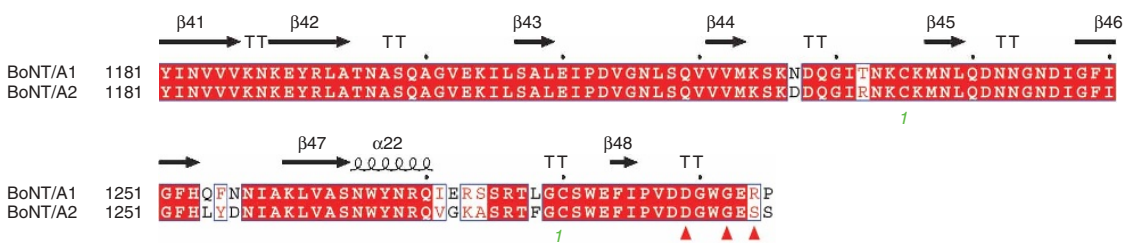

b

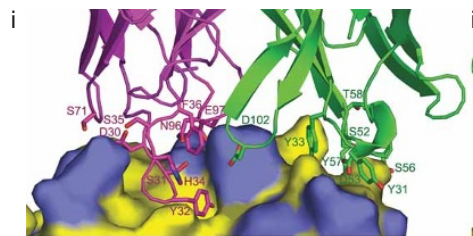

iii
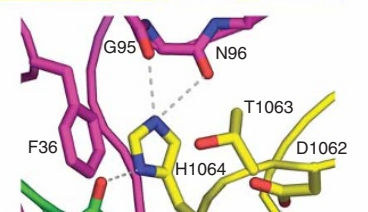

$$
\text { " }
$$

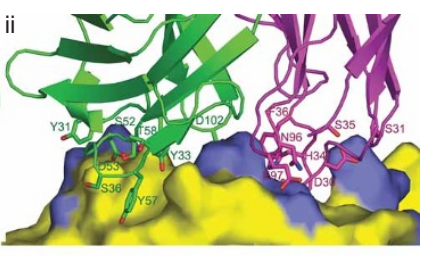

iv

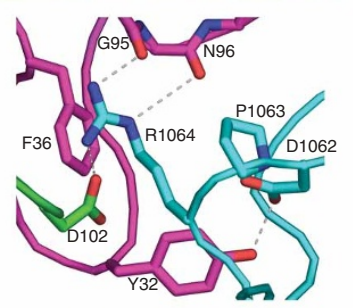

vi
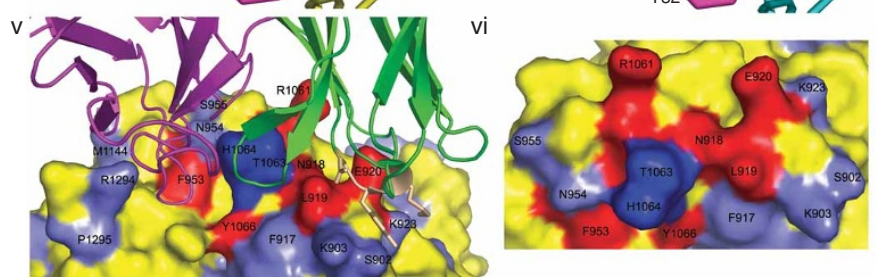

C
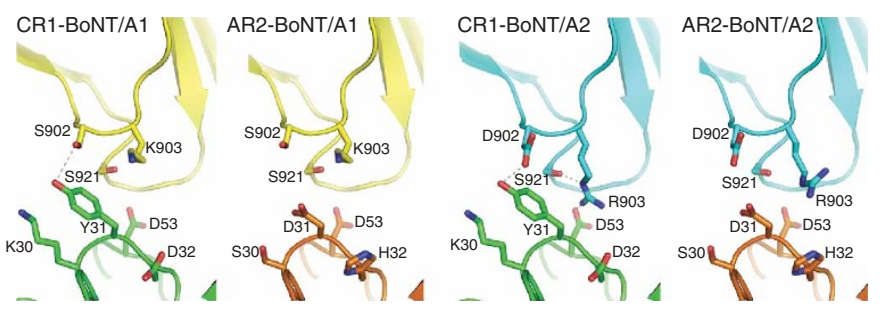

Figure 3 Location of interactions that differ between CR1 and BoNT/A1 and BoNT/A2 and affect differential BoNT/A binding. (a) Location of $\mathrm{CR} 1-\mathrm{BoNT} / \mathrm{A} 1$ contact residues and residues differing between BoNT/A1 and BoNT/A2. The alignment of BoNT/A1 and A2 subtypes shows strict sequence conservation in white letters on red background, and strong sequence conservation in red letters. The residues composing the $\mathrm{CR} 1$ epitope of the $\mathrm{H}_{\mathrm{CN}}$ lectin (residues 874-1094) and $\mathrm{H}_{\mathrm{CC}}$ trefoil (residues 1095-1295) subdomains are indicated with red triangles, energetically important residues are shown with black triangles. Disulfide bonds are indicated using green numbers. The secondary structure elements of the BoNT/A1 binding domain structure are labeled $\alpha$ ( $\alpha$-helix), $\beta$ ( $\beta$-strand) and TT (turn). (b) Structural location of differences between BoNT/A1 and A2 and impact on $C R 1$ interactions. (i,ii) Surface representations of BoNT/A1 (yellow) in complex with CR1 ( $V_{L}$ in magenta and $V_{H}$ in green) showing patches of sequence variability between BoNT/A1 and BoNT/A2 subtypes in slate blue. (iii,iv) Close-up view of sequence variability between T1063 and H0164 of BoNT/A1 (yellow, iii) and modeled P1063 and R0164 of BoNT/A2 (cyan, iv) in complex with $\mathrm{CR} 1$ ( $\mathrm{V}_{\mathrm{L}}$ in magenta and $\mathrm{V}_{\mathrm{H}}$ in green). ( $v$,vi) Surface representations of BoNT/A1 (yellow) with BoNT/A1 and BoNT/A2 sequence differences in slate blue. Key differences between BoNT/A1 and BoNT/A2 that are functionally important for binding (high $\Delta \Delta \mathrm{G}$ values) are shown in dark blue (1063 and 1064). Functionally important BoNT/A residues (high $\Delta \Delta \mathrm{G}$ values) that do not differ between BoNT/A1 and BoNT/A2 are shown in red. Panel $v$ shows CR1 with its light and heavy chains in magenta and green, respectively, with its $\mathrm{H} 1$ loop in tan. Panel vi is looking down onto the CR1 epitope of BoNT/A1 with $C R 1$ removed. (c) Details of the interaction between AR2 and CR1 and BoNT/A1 and A2 at the $\mathrm{H} 1$ loop. Close-up view of the $\mathrm{H} 1$ loop showing sequence and structural differences between BoNT/A1 (yellow) and BoNT/A2 (cyan), in complex with the CR1 (green) and AR2 (orange) Fabs. The BoNT/A2-CR1 and BoNT/A2AR2 structures are modeled.

antibody binding to BoNT/A1 (Figs. 3, 4 and Table 2). In the BoNT/A2 subtype, these residues are mutated to proline and arginine, respectively. A remarkable feature regarding CR1 cross-reactivity is that residue H1064 is deeply buried in the interface of the CR1 L3 and $\mathrm{H} 3$, forming intimate interactions with L1, L3 and H3 CDRs of CR1 (Fig. 3b (iii). Truncation of the H1064 side chain to alanine

reduces affinity of AR2 and CR1 for BoNT/A1 by more than 200,000fold (Table 2).

\section{Structural and functional basis of antibody cross-reactivity}

The identification of the BoNT/A epitope provides a means to understand the basis of engineered antibody cross-reactivity. BoNT/ $\mathrm{A} 1$ and BoNT/A2 differ by seven amino acids in the structural epitope (Fig. 3a,b and Table 2). Only two of these amino acids, T1063 and H1064 in the $\beta 35-\beta 36$, have an energetically important role in reduces the binding affinity of AR2 and CR1 for BoNT/A1 more than 200,000 -fold to $>10 \mu \mathrm{M}$. Modeling analysis, however, indicates that the H1064R mutation in BoNT/A2 would not perturb the interactions seen in the BoNT/A1 CR1 complex, as the arginine could also pi-stack with F36 and interact with D102 of H3 of CR1 (Fig. 3b (iv). This is supported by the fact that inserting the mutation H1064R into the BoNT/A1 $\mathrm{H}_{\mathrm{C}}$ reduces its affinity for AR2 and CR1 only 41- and 188fold, respectively, whereas inserting the mutation H1064A reduces its affinity for AR2 and CR1 >1,000 and >200,000 fold, respectively. 


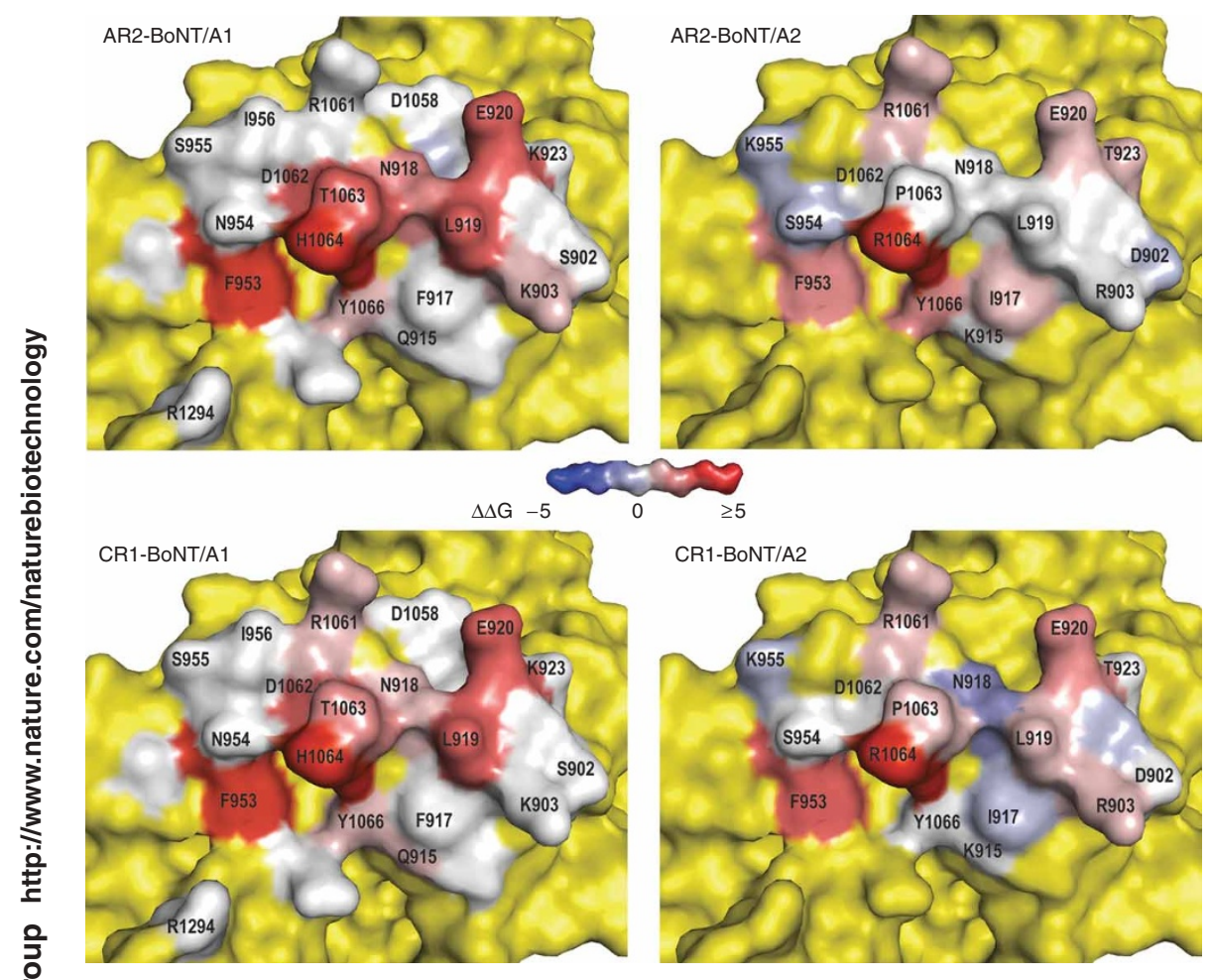

Figure 4 Energetics of binding of BoNT/A1 and BoNT/A2 to AR2 and CR1. The energetic contribution to binding of each BoNT/A1 or BoNT/A2 amino acid side chain in or near the structural epitope to AR2 and $C R 1$ was determined as described in the text and as shown in Table 2. The calculated $\Delta \Delta G$ are color coded from red to blue as shown. Amino acids contributing the greatest energy to binding are red, those with no effect are white and those whose truncation to alanine increases binding affinity are blue.

Thus, although the toxin has an amino acid substitution in the most energetically important residue in the center of the combining site, the difference can be accommodated without entirely losing antibody binding.

The engineered increase in affinity of CR1 for BoNT/A2 must result from the amino acid differences between the AR2 and CR1 H1 loops: S30K, D31Y and H32D (Fig. 3c). In this region, it must be possible to change the amino acid side chains of AR2 without losing binding affinity for BoNT/A1 in a way that picks up new binding energy for BoNT/A2. The $\mathrm{H} 1$ mutations interact with toxin residues that differ between BoNT/A1 and A2, S902D and K903R. A single interaction is observed in this region between Y31 of the CR1 H1 and S902 of BoNT/A1. The structure of AR2-BoNT/A1 reveals that the D31 of AR2 does not form this hydrogen bond with S902 of BoNT/A1. For either $\mathrm{CR} 1$ or AR2, this interaction does not contribute to the energetics of binding (Fig. 4 and Table 2). As a result, it is possible to mutate these residues in AR2 without losing binding affinity for BoNT/A1. In BoNT/A2, the hydrogen bond between CR1 Y31 and D902 of BoNT/ A2 is maintained, although this interaction still does not appear to be energetically important (Fig. 4 and Table 2). In the BoNT/A2-AR2 complex, however, D31 of AR2 would likely electrostatically clash with D902 of BoNT/A2 (Fig. 3c), contributing to a reduction in affinity for BoNT/A2. This is supported by the fact that the mutation D902A increases the affinity of AR2 for BoNT/A2 $\mathrm{H}_{\mathrm{C}}$ (Table 2, negative $\Delta \Delta \mathrm{G}$ of 0.49 ). In addition, in the CR1-BoNT/A2 interaction, an energetically important interaction appears between CR1 H1 and BoNT/A2 R903 that does not exist for AR2-BoNT/A2 (Fig. 4 and Table 2, $\Delta \Delta \mathrm{G}=+1.01$ for $\mathrm{CR} 1$ and $\mathrm{R} 903 \mathrm{~A}$ versus $\Delta \Delta \mathrm{G}=-0.02$ for AR2 and $\mathrm{R} 903 \mathrm{~A})$. The structural basis for this interaction obtained from our modeling suggests that R903 of BoNT/A2 could form a salt bridge with D53 of CR1, as well as forming additional hydrogen bonding interactions with 5921 of BoNT/A2 (Fig. 3c). The D31 of AR2 and D902 of BoNT/A2, however, would likely prevent this interaction in the AR2-BoNT/A2 complex as they are precariously close to the aliphatic end of R903 of BoNT/A2. This unfavorable interaction would be expected to result in an alternate conformation of R903. To summarize, AR2 $\mathrm{H} 1$ residues can be mutated without loss of affinity for BoNT/A1 as they do not make energetically important contacts with BoNT/A1. In CR1, mutation of these residues eliminates an electrostatics clash between AR2 and BoNT/A2 and generates a new energetic interaction with R903.

\section{DISCUSSION}

The affinity of CR2 scFv for BoNT/A2 was increased 1,250-fold while maintaining high affinity binding for wild-type BoNT/A1. The increase in cross-reactivity of CR2 equals the largest increases in affinity reported for protein-binding antibodies of a single specificity using any technique ${ }^{15,16}$. The range over which the increase in affinity for BoNT/A2 occurs (109 nM-87 pM) improves the affinity from a clinically useless value to one that would be considered high affinity for a therapeutic antibody. Only mutations in $\mathrm{H} 1$ and in the framework region led to considerable increases in crossreactivity, even though mutations were introduced into four primary antigen-binding loops ( $\mathrm{H} 1, \mathrm{H} 2, \mathrm{H} 3$ and L1). Although the increase in cross-reactivity was achieved without a crystal structure, the combination of functional studies with the co-crystal structure of CR1-BoNT/ A1 and AR2-BoNT/A1 illustrates the basis for increasing crossreactivity. Antibody amino acids tolerating mutations played no energetic role in binding to wild-type BoNT/A1; mutating these antibody amino acids led to no loss of binding to wild-type BoNT/ A1, eliminated energetically unfavorable interactions with BoNT/A2, and established new energetically favorable interactions with BoNT/ A2. This can occur only in interface areas where there are differences in antigen sequence yet no energetically important interactions between antibody and wild-type antigen. This situation may be present only in a focal region of contact between antibody and antigen and may explain why mutating amino acids in other loops, or using structure-guided mutagenesis, did not increase cross-reactivity. The mechanism for enhanced cross-reactivity described here is also different, and perhaps more specific than polyreactivity owing to conformational diversity of the antibody-combining site ${ }^{17}$ or multiple binding modes ${ }^{18}$.

Introducing mutations specifically into antigen-binding loops, as done here, has theoretical advantages over random mutagenesis. First, it allows a thorough, controlled and systematic diversification of the antibody toxin interface and permits more straightforward combination of mutations to achieve additivity ${ }^{15,16}$. Second, mutations are introduced into one of the regions of the antibody (CDRs) where mutations are naturally introduced ${ }^{19,20}$. Whereas somatic mutation also introduces mutations into the framework 
regions, the location of mutations is not random ${ }^{21}$. Random mutation may generate substitutions outside of these regions that could destabilize the antibody or increase immunogenicity ${ }^{22}$. Despite these theoretical advantages, however, CR2 resulted from mutations randomly introduced into CR1 during the PCR process. Thus there is probably a role for using random mutagenesis in a systematic approach to increasing cross-reactivity. Modeling the framework mutations of CR2 into the co-crystal structure, however, provided no insight into the mechanism by which these mutations increased cross-reactivity. In other studies, framework mutations increased affinity indirectly by affecting the equilibrium adopted among different conformational forms of the antibody ${ }^{23}$ or by precisely positioning contact residue side chains for optimal electrostatic, hydrogen bonding or van der Waals interactions ${ }^{24}$.

There are reports in which protein engineering approaches have been used to change the specificity of antibodies to haptens; however, most of these efforts have sought to narrow rather than broaden specificity ${ }^{25-28}$. Where increases in affinity for related antigens have been reported, there were considerable decreases in affinity for the cognate antigen ${ }^{29}$. Despite this, there is a therapeutic and diagnostic need for broadening antibody cross-reactivity. In the case of botulism, the existence of multiple subtypes requires antibodies with broad cross-reactivity for diagnosis and treatment ${ }^{9}$. In viral diseases, such as HIV, SARS and hepatitis B, naturally occurring mutants can cause false negative results in diagnostic immunoassays ${ }^{30}$. This work shows that dramatic increases in cross-reactivity of protein-binding antibodies can be achieved and suggests a general approach to accomplish this. In the absence of a co-crystal structure, diversity should be widely introduced to assure mutagenesis of antibody regions unimportant for wild-type antigen binding and which interact with residues that differ between antigens. Use of a highly discriminative co-selection strategy, as employed here using yeast display, is essential to assure isolation of the most cross-reactive antibodies.

The increase in cross-reactivity obtained in this study results in a more than 100-fold increase in BoNT/A2 neutralization when CR2 is combined with the human BoNT/A antibodies RAZ1 and 2G11, compared to the combination with the parental AR2 mAb (T. Smith \& J.D.M., unpublished data). As a result, the antibody generated from this study, CR2, is being manufactured along with RAZ1 and 2G11 for use as an antibody combination to treat botulism. Although formal toxicology studies have not yet been performed on the mAb combination, all three mAbs are human or humanized. Thus they should have substantially fewer side effects than equine antitoxin ${ }^{6}$. With the growing number of BoNT subtypes being discovered that have been primarily created by nature, or could be created through genetic engineering, it is critical that immune system technologies be adapted to target multiple subtypes at one time. The strategy demonstrated here provides a route for broadening the cross-reactivity of such mAbs. This approach will have substantial importance for generation of detection and therapeutic antibodies to BoNT as well as other antigens involved in disease processes.

\section{METHODS}

Oligonucleotides. Oligonucleotides for plasmid preparation for cloning sitedirected mutagenic repertoires:

H2 for SphI: 5'-GTGGTGGTGGTTCTGCTAGCGGGGCCATGGCATGG CATGCACCTACTATTCAGACAGTGTGGAGG- $3^{\prime}$

H3 for SphI: 5'-GTGGTGGTGGTTCTGCTAGCGGGGCCATGGCATGG CATGCGCTATGGACTACTGGGGCCAAGGC- $3^{\prime}$

L1 for SphI: 5'-GTGGTGGTGGTTCTGCTAGCGGGGCCATGGCATGG CATGCGGCCATAGTTTTATGCAGTGGTACC-3'
Spiked oligonucleotides for mutant library construction (synthesized by Biosource International). Spiked oligonucleotides were designed to retain either $25 \%$ or $50 \%$ wild-type amino acid at each of the five randomized amino acid positions, depending on wild-type codon usage.

H1 Rev: 5'-CTG GCG AAC CCA ATA CAT GTA 542543534521524 GAA TCC AGA G-3'

H2 Rev: 5'-TCG CCC CTC CAC ACT GTC TGA ATA GTA GGT 521542 544544 524-3

H3 Rev: 5'-CAG GGT GCC TTG GCC CCA GTA GTC CAT AGC 524524 521542 521-3'

L1 Rev: 5'-CTG CTG GTA CCA CTG CAT AAA ACT ATG GCC 521542524 514 542-3'

where

$1=70 \% \mathrm{~A}+10 \% \mathrm{~T}+10 \% \mathrm{G}+10 \% \mathrm{C}$

$2=70 \% \mathrm{~T}+10 \% \mathrm{~A}+10 \% \mathrm{G}+10 \% \mathrm{C}$

$3=70 \% \mathrm{G}+10 \% \mathrm{~T}+10 \% \mathrm{~A}+10 \% \mathrm{C}$

$4=70 \% \mathrm{C}+10 \% \mathrm{~T}+10 \% \mathrm{G}+10 \% \mathrm{~A}$

$5=50 \% \mathrm{G}+50 \% \mathrm{C}$

Oligos to amplify scFv from expression vector pYD2 (ref. 12)

pYD For: $5^{\prime}$-AGT AAC GTT TGT CAG TAA TTG C-3'

pYD Rev: $5^{\prime}$-GTC GAT TTT GTT ACA TCT ACA C- $3^{\prime}$

Gap 5': 5'-TTAAGCTTCTGCAGGCTAGTG-3'

Gap 3': 5'-GAGACCGAGGAGAGGGTTAGG-3'

Oligonucleotides for generation of $\mathrm{IgG}$ from $\mathrm{scFv}$

C25 VH5': 5'-GTACCAACGCGTGTCTTGTCCCAGGTCCAGCTGCAG GAGTCT-3'

CR2 VH5': 5'-GTACCAACGCGTGTCTTGTCCCAGGTCCAGCTGCAG CAGTCT-3'

A2B4 VH3': 5'-GTCTCCTGAGCTAGCTGAGGAGACGGTGACCAGGGT-3' Oligos for construction of yeast-displayed BoNT/A $\mathrm{H}_{\mathrm{C}}$

HC For: 5'-ATATAAACACCATGGCCACCTCCATCCTGAACCTGCGC-3'

HC Rev: 5'-TAGTATATATGCGGCCGCCAGCGGACGTTCACCCCAACC-3’

Cell lines, media, toxins and antibodies. Yeast strain EBY100 (GAL1-

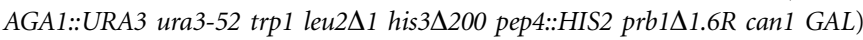
was maintained in YPD ( $1 \% \mathrm{wt} / \mathrm{vol}$ yeast extract, $2 \% \mathrm{wt} / \mathrm{vol}$ peptone, $2 \%$ $\mathrm{wt} / \mathrm{vol}$ dextrose) medium. EBY100 transfected with pYD2 was selected on selective growth dextrose casamino acids (SD-CAA) medium ( $0.1 \mathrm{M}$ sodium phosphate, $0.7 \% \mathrm{wt} / \mathrm{vol}$ yeast nitrogen base, $0.006 \% \mathrm{wt} / \mathrm{vol}$ Leucine, $2 \% \mathrm{wt} / \mathrm{vol}$ dextrose, $0.5 \%$ casamino acids). ScFv yeast surface display was induced by transferring yeast cultures from SD-CAA to selective growth galactose casamino acids (SG-CAA) medium ( $0.1 \mathrm{M}$ sodium phosphate, $0.7 \% \mathrm{wt} / \mathrm{vol}$ yeast nitrogen base, $0.006 \% \mathrm{wt} / \mathrm{vol}$ Leucine, $2 \% \mathrm{wt} / \mathrm{vol}$ galactose, $0.5 \%$ casamino acids) and grown at $20{ }^{\circ} \mathrm{C}$ for $48 \mathrm{~h}$ as described previously ${ }^{12}$. Bacteria strains Escherichia coli $\mathrm{DH} 5 \alpha,\left(\mathrm{K} 12, \Delta\right.$ (lac-pro), sup $\mathrm{E}$, thi, hsd $\mathrm{D} 5 / \mathrm{F}^{\prime} \operatorname{tra\mathrm {D}36}$, proA+B+, lacIq,

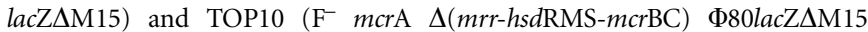
$\Delta l a c \mathrm{X} 74$ recAl araD139 $\Delta($ ara-leu $) 7697$ galU galK $r p s \mathrm{~L}\left(\mathrm{Str}^{\mathrm{R}}\right)$ endA1 nupG) were used for cloning and preparation of plasmid DNA. Pure BoNT/A1 andBoNT/A2 were purchased from Metabiologics. Recombinant purified BoNT/A binding domain $\left(\mathrm{H}_{\mathrm{C}}\right)$ was prepared as previously described ${ }^{31}$. B4 (ref. 9) and SV5 (ref. 12) antibodies were purified from Chinese hamster ovary $(\mathrm{CHO})$ cells and hybridoma supernatants, respectively, using Protein G and directly labeled with Alexa-488, using a kit provided by the manufacturer (Molecular Probes). For flow cytometry, purified IgGs 7C1 (ref. 9), 3D12 (ref. 8), B4 (ref. 9), S25 (ref. 8), and BoNT/A subtypes A1 and A2 were directly labeled with Alexa-647 using a kit provided by the manufacturer (Molecular Probes).

Creation of a library of $\mathrm{H} 1$ mutants of AR2 scFv. A spiked oligonucleotide (H1Rev) was used to introduce mutations into five amino acids located in the $\mathrm{H} 1$ loop of the AR2 scFv as previously described (Table 1) ${ }^{12}$. The oligonucleotide was designed to have a bias for $25 \%$ or $50 \%$ wild-type amino acid at each position, depending on codon usage. Library size was $2.0 \times 10^{5}$ transformants, with $27 \%$ of the unselected library expressing scFv as determined by staining with SV5 antibody and 3.65\% binding BoNT/A1.

Construction of pYD vectors for creating $\mathrm{H} 2, \mathrm{H} 3$ or $\mathrm{L} 1$ mutant libraries of CR1. Primers H2ForSphI, H3ForSphI or L1ForSphI and Gap 3' were designed 
to clone different segments of the $3^{\prime}$ end of AR2 or CR1 scFv ${ }^{32}$ into pYD2 (ref. 12) by PCR and add a $5^{\prime}$ SphI restriction site. CR1 scFv cloned into pYD2 was used as the template for 30 cycles of PCR with $50{ }^{\circ} \mathrm{C}$ annealing temperature using $P f u$ polymerase. The pYD2 vector was digested with Nco1 and NotI. Vector and one of the three PCR products were gel purified and mixed together to transform yeast EBY100 by gap repair ${ }^{12}$. The presence of the correct insert in each of the three resulting vectors (pYDH2, pYDH3 or pYDL1) was confirmed by PCR and DNA sequencing. Plasmid DNA was extracted from yeast containing each of the three plasmids and was used to transform E. coli. One Shot TOP10 chemically competent cells, following the manufacturer's instructions library construction.

Creation of libraries of $\mathrm{H} 2, \mathrm{H} 3$ or L1 mutants of CR1. Libraries of sitedirected mutants were constructed using parsimonious mutagenesis as previously described ${ }^{15,33}$. Briefly, partially degenerate primers (H2Rev, H3Rev and L1Rev) were designed to introduce mutations into five amino acids located in either the H2, H3 or L1 antigen-binding loops of the $\mathrm{scFv}$ (see Table 1) ${ }^{13,34}$ The oligonucleotides were designed to have a bias for $25 \%$ or $50 \%$ wild-type amino acid at each position, depending on codon usage. The degenerate primers and the primer Gap $5^{\prime}$ were used to amplify by PCR the corresponding $5^{\prime}$ end of the CR1 scFv gene in pYD2, and the three PCR products were gel purified. The remainder of the $\mathrm{scFv}$ gene was already cloned into pYD2 (pYD2H, pYD3H and pYD1L), as described above. Gel purified scFv gene repertoires $(5 \mu \mathrm{g})$ were combined with the appropriate vector DNA (pYD2H, pYD3H or pYD1L, $10 \mu \mathrm{g}$ digested with $N c o 1$ and Sph1) and the DNA mixtures used to transform EBY100 using gap repair ${ }^{35}$. Library sizes were 3.4, 3.6 and

윽 $4.7 \times 10^{8}$ and expression frequencies and BoNT/A1 binding frequencies were $51 \% / 8.9 \%, 53 \% / 21.8 \%$ and $62 \% / 7 \%$ for the unselected $\mathrm{H} 2, \mathrm{H} 3$ and $\mathrm{L} 1$ libraries, respectively.

Selection of mutant scFv libraries. For library selection, an amount of yeast at least ten times larger than the library size, or the sort output from the previous round, was washed and resuspended in fluorescence-activated cell sorting (FACS) buffer (PBS, pH 7.4, 1\% BSA) to which the desired concentration of pure BoNT/A was added. Selections were generally performed after allowing the reaction mixture to come to equilibrium. The volume for incubation of yeast with toxin was chosen to ensure that toxin was in at least a fivefold excess over the number of scFvs (assuming $5 \times 10^{5} \mathrm{scFv} /$ yeast $^{36}$ ). Incubation times were chosen to ensure that the reaction had come to at least $90 \%$ of equilibrium, using the formula:

$$
t=\frac{-\ln (1-\Theta)}{k_{\mathrm{on}} \bullet \mathrm{C}+k_{\mathrm{off}}}
$$

where $t=$ time, $\Theta=$ fraction of equilibrium desired (in this case 0.9 ), $k_{\mathrm{on}}=$ association rate constant, $k_{\text {off }}=$ dissociation rate constant and $\mathrm{C}=$ antigen concentration. For the calculation, the association and dissociation rate constants for AR2 IgG binding to BoNT/A1 and BoNT/A2 were used. For co-incubations with BoNT/A1 and BoNT/A2 the result giving the longer incubation time was used.

For selection of cross-reactive antibodies binding both BoNT/A1 and BoNT/ A2, BoNT/A concentrations for the five rounds of sorting the AR2 mutant library were $10 \mathrm{nM}$ BoNT/A1, $20 \mathrm{nM}$ BoNT/A2, $2 \mathrm{nM}$ BoNT/A2, $1 \mathrm{nM}$ BoNT/ $\mathrm{A} 2$ and $0.4 \mathrm{nM}$ BoNT/A2. For selection of CR1 mutant libraries, toxin concentrations for the six rounds of selection were: $1 \mathrm{nM}$ BoNT/A1, $0.4 \mathrm{nM}$ BoNT/A2, $0.1 \mathrm{nM}$ BoNT/A2, $0.05 \mathrm{nM}$ BoNT/A2, $0.025 \mathrm{nM}$ BoNT/A1 $\mathrm{H}_{\mathrm{C}}$ plus $0.025 \mathrm{nM}$ BoNT/A2 and for the final round of sorting $0.0075 \mathrm{nM}$ BoNT/A1 $\mathrm{H}_{\mathrm{C}}$ plus $0.01 \mathrm{nM}$ BoNT/A2. After incubation with toxin, cells were washed with ice-cold FACS buffer and resuspended in a 1:200 dilution of secondary antibodies as follows: (i) for BoNT/A1 or BoNT/A2 staining, 3D12-647 was added to detect toxin binding and SV5-488 was added to quantify ScFv expression; (ii) for BoNT/A2 plus BoNT/A1 $\mathrm{H}_{\mathrm{C}}$ staining, mAb B4 (binds BoNT/A1 $\mathrm{H}_{\mathrm{C}}$ ) followed by goat anti-human IgG-PE (R-Phycoerythrin $\mathrm{F}\left(\mathrm{ab}^{\prime}\right)_{2}$ from Jackson ImmunoReasearch) was used to detect BoNT/A1 binding, mAb 7C1-647 (binds BoNT/A $\mathrm{L}_{\mathrm{C}}$, not $\mathrm{H}_{\mathrm{C}}$ ) was added to detect BoNT/A2 binding, and SV5-488 was added to quantify scFv expression. Cells were incubated for 30 min with secondary antibodies at $4{ }^{\circ} \mathrm{C}$, washed once with FACS buffer, resuspended in $0.2-2 \mathrm{ml}$ of FACS buffer and sorted on a FACSAria (BectonDickinson). Typically $0.1 \%$ to $1 \%$ of yeast exhibiting both scFv expression and toxin binding were gated for collection from the single-toxin stains. For the dual-toxin stains, the brightest population for BoNT/A1 and BoNT/A2 binding was gated, the gates intersected and only yeast in both gates collected. Collected cells were grown in SD-CAA medium and used for the next round of sorting after induction in SG-CAA medium.

Measurement of yeast-displayed $s c F v$ affinity for BoNT/A. Quantitative equilibrium binding was determined using yeast-displayed $\mathrm{scFv}$ and flow cytometry as described previously ${ }^{37}$. In general, six to eight different concentrations of pure BoNT/A were used spanning a range of concentrations from ten times above to ten times below the $K_{\mathrm{D}}$. Incubation volumes and number of yeast stained were chosen to keep the number of antigen molecules in fivefold excess above the number of $\mathrm{scFv}$, assuming $5.0 \times 10^{5} \mathrm{scFv} /$ yeast. Incubation times were chosen based on anticipated times to equilibrium calculated using approximations of the anticipated association rate constant $\left(k_{\text {on }}\right)$ and dissociation rate constant $\left(k_{\text {off }}\right)$ (see above). For the higher affinity $s c F v$, this was as long as 18-24 h. Binding of BoNT/A to yeast-displayed scFv was detected using a 1:200 dilution of $1 \mathrm{mg} / \mathrm{ml} \mathrm{mAb}$ binding a nonoverlapping BoNT/A epitope (3D12) labeled with Alexa-647. To measure the antibody-toxin affinity constant $\left(K_{\mathrm{D}}\right)$ within the surface display context, only the scFv displaying yeast (SV5 binding) were included in the analysis by co-staining with SV5-Alexa-488. Each $K_{\mathrm{D}}$ was determined in triplicate, three separate inductions and measurements.

Generation of IgG from scFv. IgGs were generated from the genes encoding $\mathrm{CR} 1$ and CR2 $\mathrm{scFv}$ as previously described ${ }^{8}$. Briefly, genes encoding $\mathrm{scFv} \mathrm{V}_{\mathrm{H}}$ were amplified using PCR from their respective PYD2 vectors with the primer pairs $\mathrm{C} 25 \mathrm{VH}^{\prime}$ and $\mathrm{A} 2 \mathrm{~B} 4 \mathrm{VH}^{\prime}$ for $\mathrm{CR} 1$ and $\mathrm{CR} 2$. The $\mathrm{V}_{\mathrm{H}}$ genes from $\mathrm{CR} 1$ and CR2 were digested with $M l u \mathrm{I}$ and NheI and ligated into N5KGlcontaining the AR2 light chain variable region gene. Clones containing the correct $\mathrm{V}_{\mathrm{H}}$ were identified by DNA sequencing and vector DNA was used to transfect $\mathrm{CHO}$ DG44 cells by electroporation. Stable cell lines were established by selection in G418 and expanded into 1-liter spinner flasks. Supernatant containing IgG was collected, concentrated by ultrafiltration and purified on Protein G (Pharmacia). IgG purity was assessed by native and denaturing SDS-PAGE and concentration determined by absorbance at $280 \mathrm{~nm}$. Yields after purification were $\sim 20,25$ and $50 \mathrm{mg} / \mathrm{l}$ of culture supernatant at a cell density of $5 \times 10^{5} / \mathrm{ml}$ for AR2, CR1 and CR2, respectively.

Measurement of solution phase affinity at equilibrium. Equilibrium binding studies were conducted at $23{ }^{\circ} \mathrm{C}$ using a KinExA 3000 flow fluorimeter to quantify the free BoNT/A at equilibrium using varying concentrations of antibody. Studies of reaction mixtures were performed in PBS, $\mathrm{pH}$ 7.4, with $1 \mathrm{mg} / \mathrm{ml} \mathrm{BSA}$ and $0.02 \%$ sodium azide as a preservative. Antibody was serially diluted into a constant concentration of BoNT/A sufficient to produce a reasonable signal, where the antibody concentration was varied from less than 0.1 to greater than tenfold above the value of the apparent $K_{\mathrm{D}}$. The BoNT/A concentrations were no more than fourfold above the $K_{\mathrm{D}}$ to ensure a $K_{\mathrm{D}}$ controlled experiment. Samples were allowed to reach equilibrium for as long as $2 \mathrm{~d}$, then each of the 12 dilutions were passed over a flow cell with a $4-\mathrm{mm}$ column of Azlactone beads (Sapidyne Instruments) covalently coated with the corresponding antibody to capture the free BoNT/A. Passing an Alexa-647labeled BoNT/A antibody binding a nonoverlapping epitope over the beads produced a signal relative to the amount of free BoNT/A bound to the beads. All data points were run in duplicate and sample volume varied from 4 to $25 \mathrm{ml}$ depending on antibody affinity. The equilibrium titration data were fit to a 1:1 reversible binding model using KinExA Pro Software (Version 1.0.2; Sapidyne Instruments) to determine the $K_{\mathrm{D}}{ }^{38}$.

Measurement of solution phase kinetics. The $k_{\text {on }}$ of all antibody/antigen complexes was measured using the following 'direct method'. Antibody and BoNT/A were mixed together, then 1-2 ml of solution was passed over a freshly packed flow cell with antibody-coated beads ${ }^{39}$. Passing an Alexa-647-labeled BoNT/A antibody binding a nonoverlapping epitope over the beads produced a signal relative to the amount of free BoNT/A bound to the beads. No fewer than ten samples were sequentially analyzed, with the time between samples $\sim 500 \mathrm{~s}$. The exponential decrease in the concentration of free BoNT/A as a 
function of time was fit to a standard bimolecular rate equation using the KinExA Pro Software to determine the $k_{\text {on }}$. The $k_{\text {off }}$ was calculated from the product of $k_{\mathrm{on}} \times K_{\mathrm{D}}{ }^{38}$.

Protein expression and purification for X-ray crystallography. Fab fragments were prepared from purified IgG using immobilized papain (Pierce Biotechnology). Briefly, IgG was concentrated to $\sim 12 \mathrm{mg} / \mathrm{ml}$ in $20 \mathrm{mM}$ phosphate, 10 mM EDTA pH 7.0, then added to an equal volume of immobilized papain resin (washed with $20 \mathrm{mM}$ phosphate, $10 \mathrm{mM}$ EDTA, $20 \mathrm{mM}$ cysteine $\mathrm{pH} 7.0$ ) and incubated at $37{ }^{\circ} \mathrm{C}$ for $16 \mathrm{~h}$. The immobilized papain was removed by centrifugation at $2,000 \mathrm{~g}$, and the digest supernatant was dialyzed against $10 \mathrm{mM}$ MES pH 5.6. The Fab fragment was separated from undigested IgG and $\mathrm{F}_{\mathrm{C}}$ fragments by cation exchange chromatography (HiTrap High Performance Sulfopropyl column, GE Healthcare) using a salt gradient. The purified Fab was then dialyzed against PBS and stored at $4{ }^{\circ} \mathrm{C}$.

The ammonium sulfate-precipitated BoNT/A1 was collected by centrifugation at 16,000g and dissolved in $100 \mathrm{mM}$ HEPES, pH 7.4 and $50 \mathrm{mM} \mathrm{NaCl}$, as previously described $^{40}$. A 1:1.2 molar ratio of BoNT/A1 and CR1 Fab was mixed to form the BoNT/A1-CR1 complex followed by a final gel-filtration chromatography step. The protein complex was then concentrated to $7 \mathrm{mg} / \mathrm{ml}$ by centrifugal ultrafiltration.

Crystallization and data collection. The BoNT/A1-CR1 complex was crystallized by the nanodroplet vapor diffusion method at a temperature of $297 \mathrm{~K}$ by mixing $100 \mathrm{nl}$ of $7 \mathrm{mg} / \mathrm{ml}$ BoNT/A1-CR1 solution (10 mM HEPES pH 7.5, 50 $\mathrm{mM} \mathrm{NaCl}$ ) with $100 \mathrm{nl}$ of the reservoir solution containing 8\% PEG 20000, 8\% PEG $550 \mathrm{MME}, 200 \mathrm{mM}$ calcium acetate and $100 \mathrm{mM}$ sodium acetate at $\mathrm{pH}$ 5.5. Plate-shaped crystals were transferred to mother liquor solutions of increasing concentrations of PEG $550 \mathrm{MME}$ (up to 20\%) before harvesting and flash freezing in liquid nitrogen.

Diffraction data were collected at a wavelength of $1.000 \AA$ at the Stanford Synchrotron Radiation Laboratory (SSRL) on beam line 11-1 and at the Advanced Photon Source (APS) on beam line GM-CAT. The SSRL data set was collected at $100 \mathrm{~K}$ using an ADSC q310 CCD detector, whereas APS data set used a MARmosaic 300 detector. Data were integrated, reduced and scaled using HKL2000 (ref. 41). The BoNT/A1-CR1 crystal was indexed in the orthorhombic space group $\mathrm{P} 22_{1} 2_{1} 2_{1}$ at a resolution of $2.6 \AA$, whereas the BoNT/A1-AR2 indexed as $\mathrm{P} 2{ }_{1}$ at a resolution of $3.8 \AA$; data statistics are summarized in Supplementary Table 2 online.

Structure determination and refinement. The BoNT/A1-CR1 structure was determined by molecular replacement using the BoNT/A1 structure (PDB code 3BTA ${ }^{40}$ ) and the parent Fab structure HuC25 (S. LaPorte, R. Stroud and J.D.M., unpublished data) with $99 \%$ identity to CR1 as the search models with the program MolRep ${ }^{42}$. The BoNT/A1-CR1 model was manually built with $\mathrm{Coot}^{43}$. Structure refinement was performed using REFMAC ${ }^{44}$. The progress of the model refinement was monitored by cross-validation $\mathrm{R}_{\text {free, }}$ which was computed from a randomly assigned test set comprising $5 \%$ of the data. Refinement statistics are summarized in Supplementary Table 2 online. No electron density was observed for residues 433-452 and 623-626 of BoNT/A1. Analysis of the stereochemical quality of the model was accomplished using Molprobity ${ }^{45}$ and the AutoDepInputTool (http://deposit.pdb.org/adit/). The BoNT/A1-AR2 structure was solved using the BoNT/A1-CR1 structure as a search model. Figures were prepared with PYMOL (DeLano Scientific). Atomic coordinates and experimental structure factors of BoNT/A1-CR1 BoNT/A1-AR2 have been deposited in the Protein Data Bank with the accession numbers $2 \mathrm{NYY}$ and $2 \mathrm{NZ9}$ respectively. Residue numbering of BoNT/A1 and A2 were assigned as found in GenBank under the accession numbers AF488749 and X73423.

Sequence alignment and structural analysis. Amino acid sequences of BoNT/ A subtypes $\mathrm{A} 1$ and $\mathrm{A} 2$ were aligned using Clustal $\mathrm{W}^{46}$. A gap opening penalty of 10 and gap extension penalty of 0.2 were used with the Gonnet matrix. The alignment figure was prepared with the Clustal W alignment using ESPript using DSSP secondary structure assignments ${ }^{47}$. Structure superpositions and root-mean square deviation (r.m.s. deviation) calculations were carried out using the program $\mathrm{TOP}^{48}$. Hydrogen bonding, salt bridges and van der Waals contacts were identified with the program CONTACTS ${ }^{44}$. Buried surface areas were calculated with NOC (http://noc.ibp.ac.cn/index.htm) with a $1.4 \AA$ probe radius and standard van der Waals radii. The S902E, K903R, T1063P and H1064R changes found in BoNT/A2 were modeled by PYMOL.

Construction of yeast-displayed BoNT/A1 and BoNT/A2 $\mathrm{H}_{\mathrm{C}}$ domains. Primers HCFor and HCRev were designed to amplify a synthetic BoNT/A1 $\mathrm{H}_{\mathrm{C}}$ gene fragment by $\mathrm{PCR}^{49}$, adding the restriction sites $\mathrm{NcoI}$ and $\mathrm{NotI}$ to the $5^{\prime}$ and $3^{\prime}$ ends, respectively. The PCR product and pYD2 were digested with $\mathrm{NcoI}$ and NotI, ligated and the ligation mixture used to transform EBY100. Clones containing the correct insert were confirmed by DNA sequencing to yield pYD2/BoNT/A1 $\mathrm{H}_{\mathrm{C}}$. A BoNT/A2 $\mathrm{H}_{\mathrm{C}}$ was constructed by mutating all seven BoNT/A1 $\mathrm{H}_{\mathrm{C}}$ amino acids in the structural epitope of CR1-BoNT/A1 (S902D, F917I, N954S, S955K, S957N, T1063P and H1064R) as well as three nearby residues, K903R, Q915K and K923T, to their BoNT/A2 counterparts. This approach was chosen, rather than changing all 45 amino acids that differ between BoNT/A1 and BoNT/A2 $\mathrm{H}_{\mathrm{C}}$, to minimize the probability that the BoNT/A2 $\mathrm{H}_{\mathrm{C}}$ might not display on yeast well and to simplify construction. Construction of the BoNT/A2 $\mathrm{H}_{\mathrm{C}}$ was accomplished using PCR to introduce the BoNT/A2 residues into the relevant BoNT/A1 $\mathrm{H}_{\mathrm{C}}$ gene fragment and a combination of gap repair and restriction digestion to combine the gene fragments. Clones containing the correct insert were confirmed by DNA sequencing to yield $\mathrm{pYD} 2 / \mathrm{BoNT} / \mathrm{A} 2 \mathrm{H}_{\mathrm{C}}$.

Construction of yeast-displayed BoNT/A1 and BoNT/A2 $\mathrm{H}_{\mathrm{C}}$ alanine mutants. Genes encoding botulinum neurotoxin $\mathrm{H}_{\mathrm{C}}$ fragments in pYD2 with a single alanine substitution were constructed by using PCR and gap repair as described above for creation of BoNT/A2 $\mathrm{H}_{\mathrm{C}}$. Insertion of the correct alanine mutation was confirmed by resequencing the entire BoNT/A $\mathrm{H}_{\mathrm{C}}$ gene. After induction, mutant BoNT/A1 and BoNT/A2 $\mathrm{H}_{\mathrm{C}}$ had display levels resulting in at least a $1.5 \log$ shift when stained with SV5-Alexa-488, comparable to the levels of wild-type BoNT/A $\mathrm{H}_{\mathrm{C}}$ display.

Measurement of the affinity of CR1 and AR2 Fab fragments for yeastdisplayed BoNT/A $\mathbf{H}_{\mathrm{C}}$ mutants. The dissociation equilibrium constants $\left(K_{\mathrm{D}}\right)$ of AR2 and CR1 Fab fragments for wild-type and alanine mutants of yeastdisplayed BoNT/A1 and BoNT/A2 $\mathrm{H}_{\mathrm{C}}$ were measured by flow cytometry on a LSRII flow cytometer (BD Biosciences). First, EBY100 yeast cultures harboring the $\mathrm{pYD} 2 / \mathrm{H}_{\mathrm{C}}$ wild type or the $\mathrm{pYD} 2 / \mathrm{H}_{\mathrm{C}}$ alanine mutant plasmids were grown and induced as described above for yeast-displayed scFv. Aliquots of $\sim 1 \times 10^{5}$ induced yeast cells $\left(\sim 0.005 \mathrm{OD}_{600} \mathrm{ml}^{-1}\right)$ were washed in FACS buffer and incubated with dilutions (ranging from $15 \mu \mathrm{M}$ to $16 \mathrm{pM}$ ) of AR2 or CR1 Fab fragments such that the $K_{\mathrm{D}}$ would be spanned by at least fivefold, where possible. Incubation volumes were chosen to ensure that a tenfold molar excess of the antibody (ligand) over the displayed moiety $\left(\mathrm{H}_{\mathrm{C}}\right)$ would be maintained. For this purpose, it was assumed that $\sim 10^{5} \mathrm{H}_{\mathrm{C}}$ copies were displayed on the yeast surface. Incubation with AR2 or CR1 Fab was allowed to proceed for $4 \mathrm{~h}$ at $23{ }^{\circ} \mathrm{C}$. Cells were then washed in FACS buffer and resuspended in allophycocyanin-conjugated Fab-specific goat-anti-human $\mathrm{F}(\mathrm{ab})^{\prime}{ }_{2}$ at 1:200 dilution in FACS buffer. To accurately determine the $K_{\mathrm{D}}$ of AR2 and CR1 $\mathrm{Fab}$ fragments within the surface display context, we included only $\mathrm{H}_{\mathrm{C}}$ displaying yeast in the analysis by staining with SV5 (Alexa-488) $\mathrm{mAb}^{50}$.

Calculation of the change in free energy of binding of BoNT/A1 and BoNT/ A2 alanine mutants. For each BoNT/A1 and BoNT/A2 $\mathrm{H}_{\mathrm{C}}$ alanine mutation, the change of free energy ( $\Delta \Delta \mathrm{G}_{\text {mut-wt }}$ ) between the BoNT/A1 $\mathrm{H}_{\mathrm{C}}$ alanine (Ala) mutant relative to that of the wild type or between the BoNT/A2 $\mathrm{H}_{\mathrm{C}}$ Ala mutant relative to that of the wild-type (wt) BoNT/A2 $\mathrm{H}_{\mathrm{C}}$ was calculated using the following standard formula and using the previously measured $K_{\mathrm{D}}$ constants:

$$
\Delta \Delta \mathrm{G}_{\mathrm{mut}-\mathrm{wt}}=\mathrm{RT} \ln \left(K_{\mathrm{D}, \mathrm{Ala}} / K_{\mathrm{D}, \mathrm{wt}}\right)
$$

Note: Supplementary information is available on the Nature Biotechnology website.

\section{ACKNOWLEDGMENTS}

We thank William H. Tepp and Eric A. Johnson for providing botulinum neurotoxin. This work was partially supported by National Institute of Allergy and Infectious Diseases (NIAID) R21 grant AI53389-01 (J.D.M.), NIAID cooperative agreement U01 AI056493 (J.D.M.), Department of Defense contract 
DAMD17-03-C-0076 (J.D.M.), UC MEXUS-CONACYT Faculty Visit Fellowship (C.G.-R.) and Pacific Southwest Regional Center of Excellence grant U54 AI065359 (R.C.S.). N5KG1 was a gift of Mitch Reff, (Biogen IDEC Inc.).

\section{COMPETING INTERESTS STATEMENT}

The authors declare competing financial interests (see the Nature Biotechnology website for details).

Published online at http://www.nature.com/naturebiotechnology/

Reprints and permissions information is available online at http://npg.nature.com/

1. Centers for Disease Control. Botulism in the United States, 1899-1998. Handbook for Epidemiologists, Clinicians, and Laboratory Workers (US Department of Health and Human Services, Public Health Service, Atlanta, Georgia, 1998). 〈http://www.bt.cdc. gov/agent/botulism/index.asp $\rangle$.

2. Arnon, S.S. et al. Botulinum toxin as a biological weapon. J. Am. Med. Assoc. $\mathbf{2 8 5}$ 1059-1070 (2001)

3. United States Security Council. Tenth report of the executive committee of the special commission established by the secretary-general pursuant to paragraph 9 (b) (I) of security council resolution 687 (1991) and paragraph 3 of resolution 699 (1991) on the activities of the Special Commission Council. (United Nations Security Council, New York, 1995) 〈http://www. fas.org/news/un/iraq/s/s1995-1038.htm >.

4. Bozheyeva, G., Kunakbayev, Y. \& Yeleukenov, D. Former Soviet Biological Weapons Facilities in Kazakhstan: Past, Present, and Future (Center for Nonproliferation Studies, Monterey Institute of International Studies, Monterey, California, 1999).

5. Franz, D.R., Pitt, L.M., Clayton, M.A., Hanes, M.A. \& Rose, K.J. Efficacy of prophylactic and therapeutic administration of antitoxin for inhalation botulism in Botulinum and Tetanus Neurotoxins: Neurotransmission and Biomedical Aspects (ed. B.R. DasGupta) 473-476, (Plenum Press, New York, 1993).

6. Black, R.E. \& Gunn, R.A. Hypersensitivity reactions associated with botulinal antitoxin. Am. J. Med. 69, 567-570 (1980).

7. Amersdorfer, P. et al. Genetic and immunological comparison of anti-botulinum type A antibodies from immune and non-immune human phage libraries. Vaccine 20, 16401648 (2002).

8. Nowakowski, A. et al. Potent neutralization of botulinum neurotoxin by recombinant oligoclonal antibody. Proc. Natl. Acad. Sci. USA 99, 11346-11350 (2002).

9. Smith, T.J. et al. Sequence variation within botulinum neurotoxin serotypes impacts antibody binding and neutralization. Infect. Immun. 73, 5450-5457 (2005).

10. Willems, A., East, A.K., Lawson, P.A. \& Collins, M.D. Sequence of the gene coding for the neurotoxin of Clostridium botulinum type $A$ associated with infant botulism: comparison with other clostridial neurotoxins. Res. Microbiol. 144, 547-556 (1993).

11. Thompson, D.E. et al. The complete amino acid sequence of the Clostridium botulinum type A neurotoxin, deduced by nucleotide sequence analysis of the encoding gene. Eur. J. Biochem. 189, 73-81 (1990).

12. Razai, A. et al. Molecular evolution of antibody affinity for sensitive detection of botulinum neurotoxin type A. J. Mol. Biol. 351, 158-169 (2005).

13. Chothia, C. et al. Structural repertoire of the human VH segments. J. Mol. Biol. 227 799-817 (1992)

14. Wu, T.T. \& Kabat, E.A. An analysis of the sequences of the variable regions of Bence Jones proteins and myeloma light chains and their implications for antibody complementarity. J. Exp. Med. 132, 211-250 (1970).

15. Schier, R. et al. Isolation of picomolar affinity anti-c-erbB-2 single-chain Fv by molecular evolution of the complementarity determining regions in the center of the antibody binding site. J. Mol. Biol. 263, 551-567 (1996).

16. Yang, W-P. et al. CDR walking mutagenesis for the affinity maturation of a potent human anti-HIV-1 antibody into the picomolar range. J. Mol. Biol. 254, 392-403 (1995).

17. James, L.C., Roversi, P. \& Tawfik, D.S. Antibody multispecificity mediated by conformational diversity. Science 299, 1362-1367 (2003).

18. Sethi, D.K., Agarwal, A., Manivel, V., Rao, K.V. \& Salunke, D.M. Differential epitope positioning within the germline antibody paratope enhances promiscuity in the primary immune response. Immunity 24, 429-438 (2006).

19. Betz, A.G., Rada, C., Pannell, R., Milstein, C. \& Neuberger, M.S. Passenger transgenes reveal intrinsic specificity of the antibody hypermutation mechanism: clustering polarity, and specific hot spots. Proc. Natl. Acad. Sci. USA 90, 2385-2388 (1993).

20. Wagner, S.D., Milstein, C. \& Neuberger, M.S. Codon bias targets mutation. Nature 376 732 (1995).

21. Cumbers, S.J. et al. Generation and iterative affinity maturation of antibodies in vitro using hypermutating B-cell lines. Nat. Biotechnol. 20, 1129-1134 (2002).
22. Ewert, S., Honegger, A. \& Pluckthun, A. Stability improvement of antibodies for extracellular and intracellular applications: CDR grafting to stable frameworks and structure-based framework engineering. Methods 34, 184-199 (2004).

23. Foote, J. \& Milstein, C. Conformational isomerism and the diversity of antibodies. Proc. Natl. Acad. Sci. USA 91, 10370-10374 (1994).

24. Mian, I.S., Bradwell, A.R. \& Olson, A.J. Structure, function and properties of antibody binding sites. J. Mol. Biol. 217, 133-151 (1991).

25. Dubreuil, 0 . et al. Fine tuning of the specificity of an anti-progesterone antibody by first and second sphere residue engineering. J. Biol. Chem. 280, 24880-24887 (2005).

26. Korpimaki, T. et al. Engineering of a broad specificity antibody for simultaneous detection of 13 sulfonamides at the maximum residue level. J. Agric. Food Chem. 52, 40-47 (2004).

27. Krykbaev, R.A., Tsantili, P., Jeffrey, P.D. \& Margolies, M.N. Modifying specificity of antidigoxin antibodies using insertional mutagenesis. Protein Sci. 11, 2899-2908 (2002).

28. Miyazaki, C. et al. Changes in the specificity of antibodies by site-specific mutagenesis followed by random mutagenesis. Protein Eng. 12, 407-415 (1999).

29. Werther, W.A. et al. Humanization of an anti-lymphocyte function-associated antigen (LFA)-1 monoclonal antibody and reengineering of the humanized antibody for binding to rhesus LFA-1. J. Immunol. 157, 4986-4995 (1996).

30. Coleman, P.F. Detecting hepatitis B surface antigen mutants. Emerg. Infect. Dis. 12 198-203 (2006)

31. Byrne, M.P., Smith, T.J., Montgomery, V.A. \& Smith, L.A. Purification, potency, and efficacy of the botulinum neurotoxin type A binding domain from Pichia pastoris as a recombinant vaccine candidate. Infect. Immun. 66, 4817-4822 (1998).

32. Poul, M-A., Becerril, B., Nielsen, U.B., Morrison, P. \& Marks, J.D. Selection of internalizing human antibodies from phage libraries. J. Mol. Biol. 301, 1149-1161 (2000).

33. Schier, R. et al. Identification of functional and structural amino-acid residues by parsimonious mutagenesis. Gene 169, 147-155 (1996).

34. Tomlinson, I.M., Cox, J.P., Gherardi, E., Lesk, A.M. \& Chothia, C. The structural repertoire of the human $V_{k}$ domain. EMBO J. 14, 4628-4638 (1995).

35. Orr-Weaver, T.L. \& Szostak, J.W. Yeast recombination: the association between doublestrand gap repair and crossing-over. Proc. Natl. Acad. Sci. USA 80, 4417-4421 (1983).

36. Boder, E.T. \& Wittrup, K.D. Yeast surface display for screening combinatorial polypeptide libraries. Nat. Biotechnol. 15, 553-557 (1997)

37. Boder, E.T., Midelfort, K.S. \& Wittrup, K.D. Directed evolution of antibody fragments with monovalent femtomolar antigen-binding affinity. Proc. Natl. Acad. Sci. USA 97, 10701-10705 (2000)

38. Drake, A.W., Myszka, D.G. \& Klakamp, S.L. Characterizing high-affinity antigen/antibody complexes by kinetic- and equilibrium-based methods. Anal. Biochem. $\mathbf{3 2 8}$ 35-43 (2004)

39. Blake, R.C., II, Pavlov, A.R. \& Blake, D.A. Automated kinetic exclusion assays to quantify protein binding interactions in homogeneous solution. Anal. Biochem. 272, 123-134 (1999)

40. Lacy, D.B., Tepp, W., Cohen, A.C., DasGupta, B.R. \& Stevens, R.C. Crystal structure of botulinum neurotoxin type A and implications for toxicity. Nat. Struct. Biol. $\mathbf{5}$ 898-902 (1998)

41. Otwinowski, Z. \& Minor, W. Processing of X-ray diffraction data collected in oscillation mode. Meth. Enzymol. 276, 307-326 (1997).

42. Vagin, A. \& Teplyakov, A. MOLREP: an automated program for molecular replacement. J. Appl. Cryst. 30, 1022-1025 (1997).

43. Emsley, P. \& Cowtan, K. Coot: model-building tools for molecular graphics. Acta Crystallogr. D Biol. Crystallogr. D60, 2126-2132 (2004).

44. Collaborative Computational Project Number 4 The CCP4 suite: programs for protein crystallography. Acta Crystallogr. D Biol. Crystallogr. D50, 760-763 (1994).

45. Davis, I.W., Murray, L.W., Richardson, J.S. \& Richardson, D.C. MolProbity: structure validation and all-atom contact analysis for nucleic acids and their complexes. Nucleic Acids Res. 32, W615-W619 (2004).

46. Thompson, J.D., Higgins, D.G. \& Gibson, T.J. Clustal-W-improving the sensitivity of progressive multiple sequence alignment through sequence weighting, position-specific gap penalties and weight matrix choice. Nucleic Acids Res. 22, 4673-4680 (1994).

47. Gouet, P., Courcelle, E., Stuart, D.I. \& Metoz, F. ESPript: analysis of multiple sequence alignments in PostScript. Bioinformatics 15, 305-308 (1999).

48. Lu, G.G. TOP: a new method for protein structure comparisons and similarity searches J. Appl. Cryst. 33, 176-183 (2000).

49. Clayton, M.A., Clayton, J.M., Brown, D.R. \& Middlebrook, J.L. Protective vaccination with a recombinant fragment of Clostridium botulinum neurotoxin serotype $A$ expressed from a synthetic gene in Escherichia coli. Infect. Immun. 63, 2738-2742 (1995).

50. VanAntwerp, J.J. \& Wittrup, K.D. Fine affinity discrimination by yeast surface display and flow cytometry. Biotechnol. Prog. 16, 31-37 (2000). 